\section{False-positive serum LDH level due to interference from immunoglobulin}

Sir,

Since it became possible to measure serum enzymes in patients, we have been able to diagnose various disorders promptly. However, many substances are known to interfere with the measurement of serum enzymes. Among them enzyme-linked immunoglobulins (Ig) are sometimes reported, but in most cases the enzyme activity is relatively low, never exceeding two or three times normal values.' We report here the first case of a patient with a lactate dehydrogenase (LDH) anomaly and extremely high $\mathrm{LDH}$ levels.

A 71-year-old woman was admitted to our hospital because of a markedly elevated serum LDH level. She had suffered from mild essential hypertension and had been treated with oral antihypertensive drugs since 1990. On physical examinations, she appeared healthy and her blood pressure was well controlled. Laboratory examinations revealed that serum levels of haemoglobin, liver transaminase and creatine kinase were within normal ranges and that she did not have dysgammaglobulinaemia. However, a marked elevation of serum LDH level (reaching $3350 \mathrm{U} / 1$, especially in LDH3-5 fractions) was confirmed. A computed tomographic scan of the chest, abdomen and pelvis showed no abnormality, and endoscopic gastrointestinal examination and sigmoidoscopy revealed no evidence of malignancy. Wholebody ${ }^{67} \mathrm{Ga}$-scintigraphy did not detect any abnormalities.

To find the cause of the extremely high levels of LDH activity, we measured serum LDH after removal of Ig fractions. ${ }^{2}$ With this treatment, the $\mathrm{LDH}$ level was dramatically decreased to normal values. Enzyme immunoelectrophoresis revealed LDH bands which reacted with anti-IgA and $\kappa$-chain antiserum.

In conclusion, we should always keep in mind the possibility of an LDH anomaly in healthy patients when the LDH level does not seem to be related to the clinical state, even if the level is extremely high.

MASAYOSHI OHTA First Department of Internal Medicine SHO-ICHI YAMAGISHI Department of Biochemistry

Kanazawa University School of Medicine, Kanazawa 920, fapan

Accepted 20 August 1997

1 Taguchi H, Nishiya K, Yamanaka S, et al. Enzyme-linked immunoglobulin which binds both lactate dehydrogenase and hydroxybutyric dehydrogenase. Clin Chim Acta tyric dehydrogenas

2 Yamamoto Y, Yamagishi S, Noto Y, et al. The first case of insulin-dependent diabetes mellitus with prominent spurious hyperglucagonemia due to interference of immunoglobulin $G$ in glucagon radioimmunoassay (OAL-123) system. Hormone Res 1996;45:295-9.

\section{Delays to thrombolysis}

Sir,

I read with interest the article on minimising delays to thrombolysis in patients developing acute myocardial infarction in hospital. ${ }^{1}$ During the process of evaluation of myocardial infarct-related electrocardiographic criteria for thrombolysis, a factor which is often not taken into account is the highly variable rate of evolution of the electrocardiographic (ECG) stigmata of myocardial infarction. ${ }^{2}$ The major diagnostic challenge is to define the optimum frequency of documentation of the 12-lead ECG in those patients who are admitted with typical chest pain, but without corresponding ECG stigmata justifying thrombolysis, so that the ECG criteria for thrombolysis can be established within the therapeutic time window. According to Krucoff $e t$ al, the median interval from onset of chest pain to the ECG with the most ST segment deviation is 166 minutes, with a $25 \%$ centile value of 110 minutes, and a $75 \%$ centile value of 245 minutes. ${ }^{3}$ In practical terms, some patients can take under two hours, and others as long as four hours to manifest the typical ST segment elevation which justifies the use of thrombolytics.

OMP JOLOBE

Department of Medicine for the Elderly, Tameside General Hospital, Ashton under Lyne OL6 9RW, UK

Accepted 20 August 1997

1 Mumford AD, Banning AP. Minimising delays to thrombolysis in patients developing acute myocardial infarction in hospital. Postgrad Med myocardial infarc

2 Yusuf S, Lopez R, Maddison A, Sleight P. Variability of electrocardiographic and enzyme evolution of myocardial infarction in man. $B$ Heart f 1981;45:271-80.

3 Krucoff MW, Green CL, Trollinger KM, et al. Temporal distribution of important ECG information in patients with MI: when should you take the next ECG after lytics? (Poster 946-6). Am f Cardiol 1997;29(suppl A):131A.

\section{Arterial re-bleed following tPA administration}

Sir,

Two patients were recently admitted on to our Coronary Care Unit (CCU). The first, a 67-year-old woman, suffered from a ventricular fibrillation. She arrested shortly after admission and had electrocardiographic (ECG) changes suggestive of an anteriolateral myocardial infarction. During the arrest arterial blood gases were taken from the radial artery of the left arm. Once stabilised, the decision was taken to thrombolyse the patient, and after an adverse reaction with streptokinase, tissue plasminogen activator (tPA) was given, followed by 24 hours of intravenous heparin, successfully re-vascularising the heart. However, 24 hours post-thrombolysis she complained of a painful, swollen left forearm, which was diagnosed as compartment syndrome secondary to a re-bleed of the arterial blood gas site. She required urgent surgical decompression.

The second patient, a 78-year-old man was transferred from an acute medical ward to the CCU and had an electromechanical dissociation arrest whilst on the unit, during which arterial blood gases were taken from the left femoral artery. He was thrombolysed with tPA, followed by intravenous heparin for 24 hours, as his ECG showed changes consistent with a myocardial infarction. The following day he complained of bruising and pain in his left thigh at the site of the arterial stab, which on examination revealed a large haematoma secondary to a femoral artery re-bleed. This resolved with conservative management and was scanned with ultrasound to rule out the possibility of a false aneurysm formation

In both of these cases, the arterial blood gas sites were adequately compressed and no bleeding was noted. However, after thrombolysis with IPA and anticoagulation with heparin, the wounds re-bled, in one case with serious consequences, requiring surgery.

Re-bleed from streptokinase thrombolysis has been reported in the literature, however, we could find no reference to tPA re-bleed post-thrombolysis. We found one other case of compartment syndrome following thrombolysis, occurring in the orbit following ocular surgery' and four cases of haematoma, one following subclavian vein puncture, ${ }^{2}$ two cases of sublingual haematoma ${ }^{34}$ following oral trauma and one of facial haematoma. ${ }^{5}$

We would like to highlight the risks of tPA thrombolysis following arterial puncture and the need for vigilance in the postthrombolysis period when anticoagulating with heparin.

\section{M KNAPP \\ J E F POHL Coronary Care Unit, Leicester General Hospital, \\ Leicester, UK}

Accepted 15 September 1997

1 Marcus DM, Frederick AR Jr. Streptokinaseinduced Tenon's haemorrhage after retinal detachment surgery. $A m$ f Ophthalmol 1994;118:815-7.

2 Jorge $S$ do $C$. Reinfarction after thrombolytic therapy. Results obtained with readministration of thrombolytic agent in 3 patients (Portuguese). Arquiv Bras Cardiol 1993;60:177-82.

3 Eggers KA. Mason NP. Lingual haematoma following streptokinase therapy (letter). Anaesthesia 1994;49:922.

4 Williams PJ. Jani P. McGlashan. Lingual hae matoma following treatment with streptokinase and heparin; anaesthetic management. Anaesthesia 1994;49:417-8.

5 Jervis P, Mason JD, Jones NS. Streptokinase and facial haematoma. Postgrad Med $f$ 1995;71: 114-5. 